\title{
The shrink phenomenon
}

\author{
KENNETH S. WAGONER, FELIX E. GOODSON, and ANTONIO E. NUNEZ \\ DePauw University, Greencastle, Indiana 46135
}

Subjects were exposed to motion pictures of various objects presented at different speeds, both approaching and receding. A breakdown in size constancy occurred significantly more frequently when the signs were receding than when they were approaching and significantly more often as projection speed increased. Since such specific cues as convergence and accommodation were not activated, unless reflexively, it was inferred that a generalized perceptual set rather than the reversal of specific cues accounted for the results.

This experiment was designed to investigate an effect (the shrink phenomenon) previously unreported in the literature, demonstrating a breakdown in size constancy in an unusual perspective: the rapid recession of observed visual stimuli. Due to years of experience with visual stimuli as they move toward us, size constancy remains intact even though the retinal image literally expands as objects move closer. However, it has been observed that a visual stimulus, such as a rectangular sign, does not retain size constancy as it moves rapidly away from the observer. This breakdown in size constancy, or what we term the shrink phenomenon, can be easily demonstrated. While one is moving in a car at approximately $70 \mathrm{miles} / \mathrm{h}$, receding items such as signs and mailboxes literally shrink in one's perception.

As is well known, size constancy refers to the tendency of the human being to perceive a distant object as having the same size as a nearby one, even though the objects have different retinal projections. For example (Hilgard \& Atkinson, 1967), the retinal projection of an object at $20 \mathrm{ft}$ is only half that of the same object at $10 \mathrm{ft}$.

There is some evidence that size constancy tends to break down when the perspective is unusual. When an individual first rides in an airplane, he views the world from a vantage point that is unfamiliar, one from which the cues that have been critical in the development of his perceptual structure are, at least in part, no longer operative. He looks down and sees minute cattle grazing in diminutive fields and miniature cars running on miniature roads. In another example, reported by Turnbull (1961), a pygmy taken for the first time from the forest into open country apparently experienced a similar effect. When the pygmy saw a herd of buffalo at a great distance he asked what kind of insects they were. When told that they were buffalo, he refused to accept the fact. As he approached the herd by car, he believed that he was the victim of some sort of magic, because the buffalo kept growing in size.

Reprint requests should be sent to Felix Goodson, Department of Psychology, DePauw University, Greencastle, Indiana
46135.
The airplane example suggests that size constancy is due to central cortical structuring rather than immediately being given by experiences derived from specific cues. Indeed, many of the same cues appear to be operative when one is looking at a scene from above as when one is viewing it on the same plane. The major difference would appear to be that familiar objects vary in retinal size when viewed from the same plane, but such variation is minimal or nonexistent when the objects are viewed from above. For example, the retinal size of cars varies when they are viewed on a horizontal plane, but this does not occur when they are viewed from above, since each car is approximately at the same distance from the observer. This would suggest that variation in the size of the retinal image is a basic factor underlying the development of constancy in the perceived item and that the influence of specific cues, such as those derived from accommodation or convergence, may be unimportant.

In the following experiment, an effort was made to demonstrate the shrink phenomenon in a situation that would rule out the immediate influence of cues such as accommodation and convergence. Within a counterbalanced design, motion picutres of various objects were presented to observers at different speeds, both approaching and receding.

It was predicted that when subjects saw a motion picture of objects receding or approaching at high speed, a significantly greater number would report that objects shrink when receding than would report that objects expand when approaching.

\section{METHOD}

\section{Subjects}

One hundred and forty-seven DePauw University undergraduates of both sexes were used.

\footnotetext{
Apparatus

A 16-mm Cine-Kodak Turret movie camera, Model K-100, was used with a $25-\mathrm{mm}$ Ektar lens. Kodachrome II daylight color film was used and exposed at 16 frames/sec (fps). A variable-speed Bell \& Howell 16-mm movie projector was used to show a film at $13,16,19,22$, and 25 fps on a projection screen.
} 
Twelve objects, six painted black and six painted white, similar to common road signs were constructed of plywood. These signs were of three different shapes: square, triangle, and circle. Each shape was of two different sizes: large (36 in.) and small (24 in.). The signs were placed $7 \mathrm{ft}$ above the berm of the highway, as measured from the center of each shape. Subjects were provided an answer sheet with 120 counterbalanced alternatives.

\section{Procedure}

Filming and projection technique. Each sign was placed on the berm of a smooth highway $4 \mathrm{ft}$ from the edge of the road. The cameraman sat on the right side of a moving car, with the camera set at the normal speed of 16 fps. A motion picture was then taken, with one sequence approaching each sign and another going away from each sign, at 70-75 miles/h.

Five film copies at different speeds $(13,16,19,22$, and $25 \mathrm{fps}$ ) were made of each of these pictures, providing a total of 60 approaching trials and 60 receding trials. These 120 trials were randomly spliced together to produce the test film. After each trial there was a segment of film showing the trial number to insure that the subject would not lose his place on the answer sheet.

Instructions. As soon as the subjects were seated in the classroom, they were given a pencil and an answer sheet and were read the following instructions: "This is an experiment on size constancy. Size constancy refers to the fact that things (like cars, other people, and houses) appear to remain the same size even though the image they project on the retina is much bigger when they are close to us and much smaller when they are farther from us. For instance, if I hold this coin close to my eye, its image covers most of the retina, but if $I$ hold it at a distance, the image covers only a small part of the retina. In spite of this, this quarter seems to have the same size regardless of the distance-That is, it retains size constancy. In this experiment, we want to determine whether size constancy is affected by the speed at which things move either towards or away from us. You will see a series of moving pictures of black and white signs of different shapes and sizes. Some of these signs will be receding and some approaching your field of vision. It is your job to observe whether or not size constancy breaks down and at what speeds. At certain speeds, the signs may appear to remain the same size-that is, retain size constancy-while at other speeds, they may appear to expand or shrink. You must observe carefully and report on the answer sheet which has been handed out, by placing a check mark in the appropriate box, whether the signs appear to retain size constancy or either expand or shrink. You must make a response in every trial. If you are not sure, guess."

Following these instructions, the lights were turned down to a level that allowed the subjects to see both the answer sheet and the motion picture, and the experiment was run.

\section{RESULTS AND DISCUSSION}

The results indicated that the breakdown of size constancy occurred much more frequenctly when the signs were receding than when they were approaching. A 2 by 5 analysis of variance for a repeated-measures randomized-block factorial design (Kirk, 1968) yielded an $\mathrm{F}$ of 550.78 (significant at the .01 level). It was also found that as projection speed increased, a significantly greater amount of size constancy breakdown occurred $(F=5.43, p<.01)$.

Size, brightness, and shape were also found to be significant $(F=4.92, \quad 27.89, \quad 19.33$, respectively). Small signs provided more shrink than large, black more than white, triangles more than squares, and squares more than circles.

Differences in discriminability may have influenced these results. Perhaps it was simply more difficult to see small triangles, so that relative vagueness may have been a factor. Or perhaps it is more difficult to initially focus on a stimulus that suddenly appears and then recedes than on one that suddenly appears and then approaches. However, the magnitude of the differences obtained suggest that shrink phenomenon can be demonstrated adequately with a moving picture stimulus.

That the shrink phenomenon can be demonstrated with films, in which the observed items are always at a constant distance from the subject, further suggests that the perceptual process responsible for size constancy is of a highly generalized nature, rather than one momentarily dependent upon specific cues such as accommodation and convergence. Although such cues may contribute to the development of the process in the beginning, once it has developed, general contextual features of the visual field are apparently sufficient for its demonstration. Of course, input from the receptors activated by accommodation and convergence may still be a factor even though the peripheral stimulus conditions that normally bring about these changes are not present. That is, such changes may occur reflexively whenever the general process that may have been initially derived from them is operative.

Although different interpretations can be given of these results, an empiricistic view of perceptual organization seems most appropriate. Human beings simply have had less experience with rapidly receding than with approaching objects. The cues derived from viewing receding items have not occurred in sufficient degree for the organization underlying size constancy to occur.

\section{REFERENCES}

Hilgard, E., \& Atkinson, R. Introduction to psychology. New York: Harcourt, Brace, \& World, 1967.

KIrK, R. E. Experimental design: Procedures for the behavioral sciences. Belmont, Calif: Brooks/Cole, 1968.

Turnbull, C. M. Some observations regarding the experiences and behavior of the Bambuti Pygmies. American Journal of Psychology, 1961, 74, 304-308.

(Received for publication October 30, 1980.) 